\title{
Sociedades Anónimas Deportivas como modelo de gestión DE CLUBES: VIABILIDAD EN ARGENTINA Y LAS EXPERIENCIAS EN EL Derecho Comparado
}

\section{The Sports Limited Company as model of management of Argentina CLUBS AND THE WORLD}

Carlos Adolfo García*

Trabajo recibido el 6 de noviembre de 2018 y aprobado el 3 de junio de 2019

\begin{abstract}
Resumen
La regulación de las Sociedades Anónimas Deportivas en la Argentina es nuevamente objeto de debate, pero ahora ¿qué son estas Sociedades Anónimas Deportivas? Los tipos societarios deportivos de otros países, ¿tienen similitudes?, ¿Cuáles son los puntos controversiales en torno a su implementación?, veremos un análisis detallado de las diferentes realidades en Latinoamérica y el mundo.
\end{abstract}

Palabras clave: Sociedad Anónima Deportiva, regulación, realidades.

\begin{abstract}
The regulation of the Sports Limited Companies in The Argentina is once again under discussion, but what are the similarities? Which are the controversial issues about its implementation? The article will show a detailed study of the different realities of Latin America and the world.
\end{abstract}

Keywords: Sports Limited Companies, regulation, realities.

\section{INTRODUCCIÓN}

La importancia que han adquirido en los últimos años los clubes deportivos, aquellos en los cuales se practica una disciplina deportiva y generalmente son participantes de competiciones y/o campeonatos nacionales e internacionales refleja un tópico a estudiar por el Derecho Societario.

En vista de este panorama económico, comercial y social, el estudio del fenómeno del deporte como negocio está instalado en América Latina y la región, ya que como observaremos más adelante, en dife-

* Carlos Adolfo García. Abogado, Universidad Católica de Santa Fe- UCSF- Posadas Misiones Argentina, conferencista en Derecho Empresario y Deportivo. Ha escrito una variedad de artículos académicos en materia de negocios, deporte y Empresa. Desarrolla su actividad académica y profesional en Buenos Aires Argentina y la Provincia de Misiones, Argentina. Cursa Maestría en Derecho Empresario en la Universidad Austral de Buenos Aires, Argentina. Es Asesor Jurídico de la Honorable Cámara de Diputados de la Nación Argentina y es Socio en la Consultora GyE Consulting, Misiones Argentina. Correo de contacto: carlos_adolfog@hotmail.com 
rentes países del continente americano como Chile o Uruguay o en el continente europeo, Inglaterra o España, a pesar de sus diferencias concretas, poseen figuras societarias específicas para las organizaciones deportivas, entendiendo la especificidad del ámbito en que desarrollan su actividad.

El debate en torno a la viabilidad jurídica y económica de estos modelos de gestión se encuentra, en general, empañado de valores emocionales y de identidad con estructuras de gestión tradicionales, sin concretar profundos estudios de rentabilidad en el negocio.

El trabajo brinda un breve panorama acerca de la realidad societaria de los clubes deportivos (en general del fútbol) en la Argentina, en base a las legislaciones comparadas de países de América Latina y Europa.

\section{DePORTE, ESTRUCTURA JURÍDICA Y NEGOCIO}

El deporte a nivel internacional es un vehículo generador de grandes flujos de dinero.

Cuando hablamos de números en el deporte, es necesario destacar al fútbol, el deporte que genera mayor atracción a nivel mundial. Ante esta circunstancia, año a año los ingresos originados por derechos de televisación, por merchandising, por nombrar algunos, crecen y hacen del deporte un negocio profesional de espectáculo y entretenimiento. Por ello a continuación, vamos a analizar diferentes relevamientos de indicadores económicos de los clubes y marcas más importantes del planeta.

Según un informe anual de la Consultora Internacional KPMG del año 2018, donde se analiza las ganancias y todo lo relacionado a la rentabilidad de los clubes de las principales ligas europeas, han arrojado que la interacción en redes sociales con sus seguidores es cada vez mayor, alcanzando 111 millones de seguidores en Twitter, Facebook e Instagram (un 10\% más que el relevamiento realizado un año atrás). Si bien la cantidad de seguidores en las redes sociales no es un reflejo patente de los clubes que tienen más ingresos (el caso del Club inglés Manchester United, marca más rentable a nivel mundial, pero que se encuentra en el tercer puesto en cantidad de seguidores), si es posible aventurar que lo que percibe una institución por tener tantos seguidores no es un número menor ${ }^{1}$. Estos datos muestran una estructura de planificación y estrategia de marketing pensada en generar una marca, más allá de los resultados positivos o negativos que tenga la institución dentro del campo de juego. Si observamos la valuación de los clubes deportivos a nivel europeo, no es un detalle que entre los diez clubes mejor valuados a nivel mundial sean ingleses (Manchester United, Manchester City, Liverpool, entre otros)2. La estructura societaria, el modelo de gestión, la normativa en cuanto a derechos de imagen, la distribución de los dividendos originados por derechos de televisión y los márgenes de negocios obrantes en Inglaterra hacen que la lista de los clubes con marcas más valiosas se encuentre con varios actores de la Federación Inglesa (más adelante analizaremos la especial organización empresarial que ostentan los clubes ingleses y su impacto).

En otro informe llevado adelante por la reconocida consultora Internacional Deloitte denominado "Annual Review of Football Finance 2018", se expone nuevamente los Ilamativos valores que maneja el mercado del deporte en el marco del fútbol. Ejemplo de ello es el incremento en el ingreso colectivo del mercado del fútbol europeo de la temporada 2016/2017 que pasó de 24,6 mil millones de Euros a 26,5 mil millones de Euros, es decir un incremento de 1, 9 mil millones de Euros en relación con el período an-

\footnotetext{
KPMG (2018), p. 13.

2 KPMG (2018), p. 21.
}

GARCÍA, Carlos Adolfo. Sociedades Anónimas Deportivas como modelo de gestión de clubes: viabilidad en Argentina y las experiencias en el Derecho Comparado. Revista Justicia y Derecho, Santiago, v. 2, n 1, 2019 
terior de 2015/2016. El informe detalla que las cinco ligas más importantes de Europa (conocidas como las "top five") concentran el mayor porcentaje de estos dividendos, alcanzando el 58\% en la temporada 2016/2017, es decir unos 14,7 mil millones de Euros ${ }^{3}$. Entiéndase que las cinco ligas más importantes de Europa (España, Inglaterra, Francia, Italia y Alemania) son las que más ingresos en televisación, en transferencias de jugadores, en infraestructura e inclusive en importancia de marca como slogan de negocio tienen, por ello continúan (y probablemente continúen acentuando su poderío).

\section{Modelo de Sociedad Anónima Deportiva}

La Sociedad Anónima Deportiva (de ahora en adelante "SAD"), cuyo origen encontramos en la necesidad de profesionalizar, mercantilizar e industrializar la actividad deportiva, no solo indica un tipo social específico, sino una nueva forma de encarar los negocios del deporte desde el punto de vista económico, financiero y contable. Abordar esta cuestión ha generado opiniones cruzadas, donde los argumentos van desde la infructuosa aplicación en la práctica de las SAD, hasta el hecho de que abandonar la actual estructura que abordan los clubes es atacar una cultura tradicional Argentina ${ }^{4}$ o la región. Desde el punto de vista jurídico, y su viabilidad en la República Argentina, no es un punto menor el hecho de contemplar que la antigua tradición del país en torno a la identificación de una institución deportiva con un rol social de encuentro y cohesión de valores cercena la sana discusión en torno a una figura con evidente estructura comercial.

Mucho se ha discutido acerca de la necesidad de la modificación de la Ley General de Sociedades No 19.550 (de ahora en adelante LGS), donde se incluya entre sus tipos sociales esta nueva figura, de forma tal que la misma tenga una recepción normativa. Otros, en cambio, consideran que con la modificación del Estatuto de la Asociación del Fútbol Argentino (de ahora en adelante AFA) ya sería suficiente 5 .

Al momento de analizar de forma particular el caso del fútbol, es importante resaltar que la influencia económica no solo responde al deporte profesional, sino también a todos los sectores que participan del producto como el merchandising, marketing, turismo, publicidad, entre otros. Dicho de otra manera, los clubes contienen un gran número de interacciones con diferentes sectores, originando relaciones y vínculos jurídicos de diversa y específica índole, llegando al punto de generar enormes sumas de dinero año a año ${ }^{6}$.

\section{Antecedentes acerca de Proyectos de Sociedades Anónimas Deportivas en Argentina}

El debate en cuanto a la implementación de las Sociedades Anónimas Deportivas (de ahora en más "SAD") deviene de años de discusiones doctrinarias, exposiciones en paneles en las Jornadas Nacionales de Derecho Societario de Argentina y proyectos de leyes que han quedado varadas en meras intenciones.

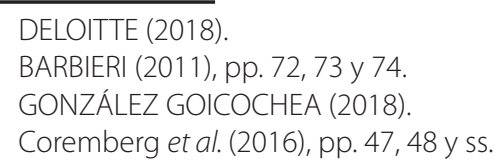

GARCÍA, Carlos Adolfo. Sociedades Anónimas Deportivas como modelo de gestión de clubes: viabilidad en Argentina y las experiencias en el Derecho Comparado. Revista Justicia y Derecho, Santiago, v. 2, n 1, 2019 
El antecedente más interesante fue el Proyecto de Ley en el Congreso de la Nación Argentina del año 2000, elaborado por el senador tucumano José Yayo Carbonell. Dicha iniciativa establecía en su artículo primero: "Las asociaciones o entidades civiles autorizadas a funcionar como personas jurídicas podrán desarrollar una o más disciplinas deportivas de carácter profesional a través de una Sociedad Anónima Deportiva (SAD), la que se constituirá con terceros a ese efecto. Dichas Sociedades Anónimas Deportivas se sujetarán al régimen general de la Ley de Sociedades Comerciales 19.550 y sus modificatorias..."7. Como se observa, había una clara remisión al régimen general de la LGS Argentina, como normativa de aplicación directa (ya que aquel proyecto de ley tenía tan solo 39 artículos, y su desarrollo no contemplaba todos los institutos propios del derecho societario).

El proyecto presentado por Carbonell, que perdió estado parlamentario en el año 2002, contenía especiales consideraciones en torno a salvaguardar los intereses de los asociados a las instituciones deportivas de la Argentina, como en el caso de diferenciar el gerenciamiento de las actividades profesionales, es decir, las disciplinas deportivas profesionales, derechos de marca, televisión, sponsors, entre otros, quedaban en la órbita del tipo societario comercial, en cambio todo lo ateniente a actividades sociales, de recreación, deportes amateurs, entre otras cuestiones, eran reservadas a la figura societaria sin fines de lucro, "... la constitución de una sociedad anónima deportiva por la asociación civil con terceros, no implica una transformación, pues ésta solamente tendrá a su cargo el desarrollo de una o más disciplinas deportivas de carácter profesional, dejando para la asociación civil el cumplimiento de sus restantes actividades" ${ }^{\prime \prime}$.

En cuanto a la disposición accionaria de las SAD, el artículo № 10 del mencionad proyecto, establecía que podían ser accionistas "... personas físicas o jurídicas argentinas y personas físicas o jurídicas extranjeras con domicilio en la República que no superen cada una de ellas el 25 por ciento del capital social". La interpretación literal del proyecto permite elevar una primera conclusión: hay una decisión de que los capitales extranjeros ingresen al cuerpo accionario de un club deportivo, en vistas de alentar en primer lugar el ingreso de capitales y en segundo lugar la inversión profesional en la gestión de organismos del deporte. Como contrapartida, la iniciativa preveía la asistencia de un conjunto de organismos y departamentos creados a especial efecto de la fiscalización, control, desarrollo y manejo de todo lo ateniente a las SAD compuesto por un Departamento Especial de Sociedades Anónimas Deportivas para que funcionara en la IGJ con el asesoramiento de ambas Cámaras del Congreso, la Secretaría de Deporte, la Confederación Argentina de Deportes, el Comité Olímpico Argentino y la AFA?

Se puede observar que la autoridad de aplicación en materia de Sociedades en Capital Federal -IGJcontenía una especial asistencia de un órgano legislativo, un órgano del Poder Ejecutivo y finalmente la colaboración de dos Personas Jurídicas Privadas netamente deportivas, dicho esto, considero atinado y de buen criterio la implementación de este cuerpo, con efectiva respuesta a paliar las vicisitudes probables de la puesta en marcha de un sistema extraño hasta el momento.

\section{5.- La Figura Asociativa Argentina en el Deporte y sus particularidades}

En la República Argentina, la figura jurídica utilizada por los clubes deportivos en general es la Asociación Civil sin Fines de lucro (de ahora en adelante ACSFL) contenidas en el Código Civil y Comercial

\footnotetext{
7 VEIGA (2016).

8 NISSEN (2000), p. 380.

9 VEIGA (2016).
}

GARCÍA, Carlos Adolfo. Sociedades Anónimas Deportivas como modelo de gestión de clubes: viabilidad en Argentina y las experiencias en el Derecho Comparado. Revista Justicia y Derecho, Santiago, v. 2, n 1, 2019 
como personas jurídicas de carácter privado, los cuales no pueden perseguir el lucro como fin principal para sí o para terceros ${ }^{10}$. Esto ocurre debido a que a principios del siglo pasado, el hecho de asociarse y constituir un club deportivo en ninguna manera significaba obtener ganancias, pues no había un mercado profesional, el objetivo claro era fomentar polos de encuentro social, cuestión que entiendo debe ser estudiada a fin de que la institución deportiva no sufra contratiempos en su competición deportiva-empresaria11. Hoy esta figura presenta ciertos desacoples estructurales, ya que la incorrecta e ineficiente administración de las entidades, inclusive sospechadas de corrupción, menoscaban la fortaleza y confianza que este modelo de estructura deportiva tenía años atrás (los casos de Racing Club de Avellaneda, que, tras la suspensión de pagos, fue intervenido judicialmente ${ }^{12}$ y Colón de Santa Fe en similar circunstancia operativa).

Las transacciones que llevan adelante los clubes o instituciones deportivas generalmente contienen grandes sumas de dinero, ejemplo de ello son la compraventa de jugadores, lo cuales a criterio del Sistema de Contabilidad Nacional del 2008 (de ahora en adelante "SCN08) los ingresos por dichas transacciones no constituyen producción, ya que los jugadores son considerados como "activos no producidos"13. Dicha concepción restrictiva no es coincidente con la idea de que los jugadores son el principal activo intangible del que disponen los clubes, y su producido marca la sostenibilidad y bienestar futuro de la organización. Por otro lado, se encuentra en total disconformidad con los motivos fundantes de institutos que buscan beneficiar a clubes formadores de deportistas del Derecho del Deporte como la Indemnización por Formación y Mecanismo de Solidaridad en el fútbol ${ }^{14}$, en vistas de que la formación integral de un deportista demanda una erogación al club.

En la Argentina los clubes de fútbol se encuentran bajo la órbita de la AFA como autoridad de aplicación, la cual dispone en su Estatuto Constitutivo que la inscripción como miembro debe ser mediante la estructura de una ACSFL, por ello pareciera jurídicamente inviable la presencia de una Sociedad Anónima Deportiva, al menos desde la exigencia para competir ${ }^{15}$.

Considero que no es posible condicionar la competición de un club en torneos oficiales locales con la eminente obligación reglamentaria a que sean entidades sin fines de lucro. Esto, más allá de las facultades delegadas a un organismo nacional que nuclea a los clubes, debería ser al menos observado y debatido con la participación e los asociados directos e indirectos a la AFA, concretando un debate federal, donde se expongan argumentos solventes en favor y en contra. La naturaleza del fútbol, exige que se profesionalice los sectores, sobre todo en un mercado donde los volúmenes y márgenes de ingresos son enormes, como se detalló oportunamente.

\section{Regulación de Sociedades Anónimas Deportivas en Latinoamérica}

A continuación, exponemos algunas de las principales normativas y realidades en torno a las figuras societarias como modelo de gerenciamiento de clubes o instituciones deportivas. Si bien existen ordenamientos que no podremos estudiar en este ensayo por razones de orden metodológico (como ser la normativa de Brasil -9615/98- o el ordenamiento alemán), la realidad marca que la necesidad de atraer

10 Código Civil y Comercial de La Nación Argentina, edición 2015.

11 BARBIERI (2011), pp. 214 y 215.

12 FRANCISCO VICTORIA ANDREU (2012), pp. 10 y 11.

13 COREMBERG et al. (2016), pp. 52 y ss.

14 FEDERACION INTERNACIONAL DE FÚTBOL ASOCIADOS (2018), p. 26.

15 ASOCIACIÓN DEL FÚTBOL ARGENTINO, Estatuto. (2017).

GARCÍA, Carlos Adolfo. Sociedades Anónimas Deportivas como modelo de gestión de clubes: viabilidad en Argentina y las experiencias en el Derecho Comparado. Revista Justicia y Derecho, Santiago, v. 2, n 1, 2019 
mayores flujos económicos, diversificar los ingresos y profesionalizar la gestión de los clubes deportivos ha volcado en Sudamérica una corriente de debate e implementación de estos modelos societarios específicos.

En Chile, existe la Ley Nacional № 20.019 que regula las Sociedades Anónimas Deportivas Profesionales (de ahora en adelante "SADP"), vigente desde mayo del año de 2005. Dicha normativa surgió luego de la quiebra de Colo Colo FC en 2002 y Universidad de Chile en el año 2006. Esta ley es de carácter especial, y la ley de Sociedades Anónimas No 18.046 sancionada en 1981 funciona como normativa subsidiaria (así lo expresan los artículos 5, 12, 15, entre otros).

La normativa del país trasandino define a la SADP como: “. . Son organizaciones deportivas profesionales aquellas constituidas en conformidad a esta ley, que tengan por objeto organizar, producir, comercializar y participar en espectáculos deportivos y que se encuentren incorporadas en el registro...", continúa la norma estableciendo que las organizaciones deportivas profesionales podrán tener el formato de corporaciones, federaciones o Sociedades Anónimas.

La Ley establece expresas exigencias en cuanto a la obligatoriedad de llevar libros, balances anuales, estar al día en cuanto a las obligaciones sociales y previsionales con sus trabajadores (artículos 6 y ss.). Por otro lado, se establece un capital social mínimo fijado en mil unidades de fomento (se establece un plazo mínimo de un año para concretar este monto contado desde la fecha de aprobación de la asamblea que da origen a la Sociedad Anónima, funciona como una condición resolutoria).

Debemos mencionar que la transformación social a Mercantil existente en los clubes, no alcanza a las Federaciones Deportivas Nacionales, quienes conservan su carácter de Asociaciones Civiles, es decir, que aquellos entes que organicen y nucleen clubes en pos de organizar competencias y reglamentar el deporte dentro del territorio chileno no deberán transformar su organización. También se incluye un capítulo completo dedicado a la fiscalización el cual corresponde a la Superintendencia de Valores y Seguros ${ }^{16}$.

Colombia también posee disposiciones en torno a las SAD en la Ley Nacional No 181 del año 1995 y la ley No 1445 que la modifica e introduce la posibilidad que los clubes con deportistas profesionales deben optar por estructurarse como Corporaciones u Asociaciones del Código de Civil o bien Sociedades Anónimas del Código de Comercio. En la mencionada norma que introduce reformas e incluye a las SAD, se establece un mínimo de cinco socios (número mayor que en la legislación de Chile, recordemos que eran 3 mínimo) y un mínimo de capital suscripto para las SAD de disciplinas deportivas diferentes al fútbol a la fecha de constitución que es de cien salarios mínimos (el salario mínimo actual es de \$781,242.00) ${ }^{17}$ y en el caso que la disciplina profesional sea el fútbol el margen mínimo es de mil salarios mínimos, si por algún motivo la SAD quedara con un capital inferior a los mencionados, se revocará su "reconocimiento deportivo" acorde a la redacción del artículo № 4.

Otro punto interesante de la normativa son los estrictos controles que recaen sobre los aportes de capitales a las SAD (control ejercido por el Instituto Colombiano de Deportes, quien tiene la facultad de solicitar información a entidades públicas y privadas acerca del origen de los fondos); En cuando al control de los movimientos de los futbolistas profesionales (ventas, cesiones, etcétera) se establecen estrictas fiscalizaciones a través de la Unidad de Información y Análisis Financiero de Colombia, quien podrá requerir determinados reportes para validar el origen de los fondos, la correlación de capacidades económicas entre los accionistas, etcétera.

16 Ley № 20.019 de 2005.

17 Página web Oficial Salario Mínimo Colombia (2018).

GARCÍA, Carlos Adolfo. Sociedades Anónimas Deportivas como modelo de gestión de clubes: viabilidad en Argentina y las experiencias en el Derecho Comparado. Revista Justicia y Derecho, Santiago, v. 2, n 1, 2019 
Además, se establecen estrictos procedimientos de conversión de los clubes con deportistas profesionales (cualquiera sea su estructura de organización) a SAD, debiendo cumplir con exigencias de aprobación de Asamblea, controles y fiscalizaciones ${ }^{18}$.

La realidad operativa de los clubes antes la posibilidad de Conversión a SAD en Colombia se ve respaldada por número operativos deficitario en los clubes. Un ejemplo de ello es el Club Millonarios (actualmente Millonarios S.A.), el cual estuvo al borde de la liquidación con un pasivo que alcanzaba los 17 millones de dólares en el año 2001 hasta que logra conseguir inversionistas por 13 millones de dólares. Luego de obtener el capital mencionado y reunir la cantidad de socios que exigía la ley, surge la Sociedad Azul y Blanco S.A. Gracias a la excelente gestión, el club comenzó a ser protagonista en las competiciones y en 2012 se corona campeón de la Copa Postobón y logra tener estabilidad financiera19 ${ }^{19}$

En el año 2010, Perú sancionó la Ley No 29.504, denominada: "Ley que promueve la transformación y participación de los Clubes Deportivos de fútbol profesional en Sociedades Anónimas Abiertas". Esta normativa con el objetivo de "promover su gestión en términos de transparencia y eficiencia, para hacer más competitiva y de alto rendimiento esta disciplina deportiva" establece la posibilidad de gerenciar los clubes deportivos a través de figuras societarias comerciales o a través de diferentes maneras: a) La participación en sociedad anónima abierta; b) Celebrar un contrato de gerencia con una empresa para la administración del Fondo de Deporte Profesional que constituirá, conservando la personería jurídica de asociación; c) Celebrar un contrato de concesión privada. Las opciones que establece la norma son ante el caso de que un club deportivo no adopte un tipo societario (es decir, conserve su estructura de asociación).

Como breve comentario, me parece muy acertada la mecánica establecida por la normativa, en torno a facilitar el "salvamento" de los clubes a través del contrato de cisión privada para aquellos que "...se encuentren en estado de insolvencia declarado o no... para entregar al concesionario el uso y goce de todos sus bienes, corporales e incorporales, incluidos los derechos asociativos y federativos, el que continuará con la actividad deportiva profesional haciendo uso de su propia personería jurídica...", es decir, se permite que el club continúe en el desarrollo normal de sus actividades, y que su estado financiero no cercene su capacidad deportiva.

En este marco, es de destacar que aquellos clubes que conserven su estructura de asociación civil, formarán el Fondo del Deporte del Fútbol Profesional destinado a financiar las diferentes erogaciones que signifiquen participar de los torneos organizados a nivel doméstico y aquellos de FIFA (Federación Internacional de Fútbol Asociados). Este fondo, de administración independiente, tiene el objetivo de paliar las deficiencias económicas que presentan las asociaciones deportivas, en cuanto a que poseen inmensos pasivos y muchas veces los ingresos no los cubren.

Otro punto interesante del modelo peruano es su control, ya que se sienta un control coordinado entre El Instituto Peruano del Deporte (IPD) y la Comisión Nacional Supervisora de Empresas y Valores (Conasev) de forma oficiar de entes de control del cumplimiento de la presente normativa y sus reglamentaciones ${ }^{20}$.

En el año 2001, Uruguay sanciona la ley No 17.292 en su sección XII Título I establece la regulación de las SAD. La norma (reglamentada por el Decreto № 223/001) establece que los clubes podrán adoptar (opcional) la estructura de Asociaciones Civiles o SAD (quienes adopten esta estructura, se someterán al régimen general de Sociedades Comerciales de Uruguay).

18 CONGRESO DE LA REPUBliCA DE COLOMBIA (2011): Ley Nacional de Colombia No 1445. Arts. 1, 3, 5, 8, 9, 10 y ss.

19 Trujillo Cabrera (2014), pp. 9 y ss.

20 Ley № 29.504 (21.01.2010), Lima, Perú. Ley que promueve la transformación y participación de los Clubes Deportivos de fútbol profesional en Sociedades Anónimas Abiertas.

GARCÍA, Carlos Adolfo. Sociedades Anónimas Deportivas como modelo de gestión de clubes: viabilidad en Argentina y las experiencias en el Derecho Comparado. Revista Justicia y Derecho, Santiago, v. 2, n 1, 2019 
Del análisis de los textos del artículo № 67 y 70 surge que no hay impedimento para que los clubes adopten cualquier forma jurídica admitida en el ordenamiento jurídico uruguayo, siempre que respondiera a la finalidad específica que persigue la norma²1.

A diferencia de las legislaciones de Chile y Perú (donde se establecen especiales capitales mínimos para constituir una SAD), la normativa uruguaya remite al ordenamiento societario común para una Sociedad Anónima²2.

\section{INGLATERRA, UN MODELO CORPORATIVO Y PROFESIONAL}

Inglaterra, el primer país en adoptar estructuras privadas sus clubes (desde el año 1921), siendo el Tottenham Hotspurs el primero en cotizar en la Bolsa de Londres (London Stock Exchange) inserta los argumentos por los cuales los clubes ingleses poseen gran desarrollo estructural, tanto en la Championship (segunda división del fútbol de Inglaterra) como en la Premier League (primera división).

Hoy en día, la Federación Inglesa (en inglés "Federal Association") no exige una estructura Jurídico Comercial determinada de organización de un club, pero si establece que no pueden los clubes constituirse como empresarios individuales (Sole Trader). Los Tipos Comerciales más usados son a) unincorporated association (Asoc. Civil sin Personalidad Jurídica) es la estructura más común y más usada por los clubes amateurs, debido a sus bajos costos y su facilidad de constitución. Entre las principales características que se pueden destacar de esta figura es de que no constituye una persona separada de sus miembros (ante reclamos patrimoniales por indemnizaciones u otros conceptos, los miembros del club podrían responder con su patrimonio). Otra nota distintiva es la flexibilidad en cuanto a las exigencias legales, en comparación con las Limited Companies; b) private company limited by guarantee (Soc. Limitada de Resp. por Garantía: Es el tipo recomendado para aquellos proyectos fundados sin intención de obtener ganancias para sus miembros, pero constituidos bajo un modelo de capital. Es la estructura más usada por las organizaciones de voluntariado u ONG's (Organizaciones no Gubernamentales). El detalle está en que es una persona jurídica independiente de sus miembros, pues ellos responderán hasta la medida de sus aportes. La excesiva publicidad de su funcionamiento (registro de miembros, de directores, etcétera) en conjunto con la imposibilidad de transferir su calidad de miembro aun tercero y el hecho de no tener ganancias hacen que el modelo no tenga demasiada utilidad en el deporte; c) private company limited by shares (Soc. Limitada por acciones). Es el modelo ideal para las instituciones deportivas que buscan una personalidad diferenciada de la de sus miembros, pero que además no buscan operar como entidades sin ánimo de lucro (permite la distribución de dividendos y ganancias en caso de liquidación y disolución, mecanismos no receptados por el modelo del punto "b"). La estructura es similar a la private company limited by guarantee con la diferencia que en este caso sus accionistas eligen a sus directores. Otro atractivo del modelo es la libertad de transferencia (compra y venta) de las acciones por parte de los accionistas, sin límite alguno. Se prevén porcentajes de tenencia accionaria que permiten el control de la compañía (50\% para remover a directores y 75\% modificación de artículos); d) Community Interest Company (Compañías de Interés Comunitario): Son Sociedades Anónimas las cuales persiguen el desarrollo de un negocio o actividad en beneficio de intereses comunes de la comunidad, sin tener ventajas privadas. Es una figura con personalidad separada de la de sus miembros, por los cuales se ven protegidos de responsabilidades que contraiga la compañía. Una ventaja llamativa del modelo es su rápida constitución (en un plazo de

21 OLIVERA GARCÍA (2001), pp. 15, 16 y ss.

22 Ley No 17.292. (29.01.2001) Montevideo, Uruguay. "Administración Pública, Empleo, Fomento y Mejoras".

GARCÍA, Carlos Adolfo. Sociedades Anónimas Deportivas como modelo de gestión de clubes: viabilidad en Argentina y las experiencias en el Derecho Comparado. Revista Justicia y Derecho, Santiago, v. 2, n 1, 2019 
cinco días hábiles). Un punto en contra es que no recibe beneficios fiscales ni tributarios, como si perciben las Private company limited by guarantee. Debido a su complejidad al momento de administrarla, su creciente control por autoridades públicas y su desconocimiento por el público en general, no ha tenido mayor éxito en la realidad; e) Co-operative or Community Benefit Society (similares a las Cooperativas en el Derecho Argentino y Sudamericano ): Entre sus detalles se encuentra que es una sociedad cooperativa formada en beneficio de sus propios miembros únicamente y en definitiva un negocio administrado en beneficio de la sociedad en general. Posee una estructura democrática que otorga un voto a cada miembro. Una ventaja significativa en el marco del mercado es que no posee límite (salvo especial disposición en contrario) en cuanto a la emisión de acciones en cabeza de sus miembros. Permite la oferta pública de membresías, lo que posibilita el mayor y más rápido acceso a capitales externos. Las ganancias no deben ser distribuidas entre los miembros, sino que deben ser destinadas a fortalecer el objetivo social. Las principales desventajas se encuentran en el burocrático sistema para obtener habilitación y registro (demostrar especiales razones que demuestren el indicado uso de este tipo social y no otro; elevadas tasas de registro y trámites; etcétera), en limitaciones de inversión que recaen en sus diferentes tipos de miembros y la posibilidad de que algunos miembros (withdrawable shares) pueden retirarse y la Persona Jurídica debe reintegrar su aporte (lo cual genera una incertidumbre e inestabilidad en la estructura económica del sistema) y f) Charitable Incorporated Organisations: Modelo incorporado en 2013, con el objetivo de canalizar a aquellas instituciones que buscan operar únicamente como Sociedad Benéfica legalmente constituida, pero sin estar bajo la legislación corporativa inglesa. Pues en este tipo social, al no ser una compañía no está bajo el control de la Cámara de Empresas de Inglaterra (Companies house) si por la Comisión de Caridad. Tienen amplios beneficios impositivos y flexibilidad en adopción de estructura interna. Al no existir un registro público de los gastos de estas entidades, en la práctica es difícil concretar contratos crediticios u obtener préstamos bancarios; muchas veces el hecho de no tener la aprobación de la Comisión de Caridad (y por ende su existencia legal plena) dificulta el funcionamiento del ente ${ }^{23}$.

Es interesante destacar que el amplio desarrollo en materia corporativa y de Personas jurídicas en Inglaterra responde a una organización interna del fútbol inglés, no solo a través de organismos públicos registrales, el fisco o reparticiones públicas, sino por su propio diagrama federativo disciplinario-regulador.

El organigrama que sostiene un mercado deportivo, donde los clubes, en general, con amplio margen económico y que cotizan en bolsa (más allá de los órganos públicos de control por parte del Estado) se compone de: The Federal Association (De ahora en adelante "FA") - quien se ocupa de organizar y oficiar de regulador de las competencias de fútbol, la FA Board (compuesto por varios miembros y uno de ellos independiente - The Charmian-, quienes tienen la función de asegurar el efectivo gobierno del juego por parte de la FA), FA Council (Consejo que funciona como un "parlamento del fútbol", y se ocupa en mayor medida de velar que el funcionamiento de la FA Board sea en "interés del fútbol") y Football Regulatory Authority (FRA, cuerpo semi autónomo con la principal función de controlar que el desarrollo de la potestad normativa de la FA este correctamente aplicada) ${ }^{24}$. No es común que el organigrama deportivo tenga este tipo de estructuras de control privadas (federativas) interrelacionado, donde el poder disciplinario a los organismos de primer grado (clubes) es extremadamente riguroso, debido a que los cuerpos disciplinarios y aquellos encargados de sancionar circunstancias deportivas se ven en constante evaluación y fiscalización por otras instituciones (es el caso de la Football Regulatory Authority, pues su independencia solidifica su capacidad y criterio de control).

23 The Federal Association (2015), pp. 6-16. Traducción de mi propia autoría.

24 All Party Parliamentary, Football Group (2009), pp. 05-15. Traducción de mi propia autoría.

GARCÍA, Carlos Adolfo. Sociedades Anónimas Deportivas como modelo de gestión de clubes: viabilidad en Argentina y las experiencias en el Derecho Comparado. Revista Justicia y Derecho, Santiago, v. 2, n 1, 2019 


\section{Sociedades Anónimas Deportivas, experiencia en algunos países de Europa}

A continuación, se desarrolla un breve análisis acerca de la figura comercial específica en el deporte receptado en España, Italia y Francia.

\subsection{Las Sociedades Anónimas Deportivas en España}

En España existe la Ley No 10/1990 y el Real Decreto 1084/1991 que establece el modelo de las Sociedades Anónimas Deportivas. Dichas normas establecen que en principio la transformación se produce "por efecto de la ley", una vez cumplido el proceso establecido, salvo exista saldo patrimonial neto positivo en los últimos cuatro períodos. Los clubes que no se transformen o adscriban sus equipos profesionales, no podrán participar en competiciones profesionales. Esta legislación fue implementada como remedio a las experiencias financieras desfavorables que los clubes españoles enfrentaban, entendiendo que un mayor intervencionismo estatal sería provechoso ${ }^{25}$. De esta manera se buscaba llevar más transparencia al deporte y concretar un antiguo anhelo de generar mayores ingresos a través de la diversificación de recursos.

En este marco, se han generado una serie de controversias en la Comisión Europea la cual ha estado abriendo expedientes en los últimos años para investigar supuestos de presuntas infracciones a las reglas comunitarias reguladoras de la libre competencia a las entidades españolas que no han adoptado el formato de SAD y se han mantenido como clubes deportivos, pero de base asociativa y han estado percibiendo ayudas económicas de carácter público ${ }^{26}$.

\subsection{Modelo de Sociedad Anónima Deportiva Italiana}

En el régimen normativo italiano, se encuentra vigente el Real Decreto 1251/1999 que legisla las Sociedades Anónimas Deportivas. La misma establece que podrán contratar atletas profesionales únicamente las sociedades deportivas constituidas bajo la forma de sociedades por acciones o sociedades de responsabilidad limitada. En este orden, la sociedad debe desarrollar de forma exclusiva actividades deportivas o conexas. La misma debe destinar obligatoriamente 10\% de sus ganancias (mínimo) a escuelas juveniles de adiestramiento y formación técnico-deportiva (se establece un fin social en su gerenciamiento). La sociedad deberá estar afiliada a una o más federaciones nacionales reconocidas por el Comité Olímpico Nacional italiano. Asimismo, la ley impone la designación de una comisión fiscalizadora para todas las sociedades deportivas a fin de efectivizar el control sobre estos tipos societarios ${ }^{27}$.

\subsection{Francia y un enfoque Corporativo-Social de clubes}

En orden a mencionar con claridad cuál es la variedad en torno a los tipos sociales existentes en Francia, debemos mencionar que existen tres tipos de Sociedades Comerciales que los clubes pueden adoptar: a) Sociedad deportiva unipersonal a responsabilidad limitada: ha tenido poco éxito. b) Sociedad anónima con objeto deportivo (SAOS): Sociedad sin fines de lucro y c) Sociedad anónima deportiva profesional (SADP).

\footnotetext{
25 MENÉNDEZ et al. (1997), pp. 134 y ss.

26 DELGADO TRUYUOLS (2014).

27 Victoria-Andreu (2012), pp. 4 y ss.
}

GARCÍA, Carlos Adolfo. Sociedades Anónimas Deportivas como modelo de gestión de clubes: viabilidad en Argentina y las experiencias en el Derecho Comparado. Revista Justicia y Derecho, Santiago, v. 2, n 1, 2019 
Lo más interesante del modelo francés es la convivencia armónica que existe entre dos modelos de gestión, ya que la Sociedad Anónima que se crea, se acopla a la asociación de origen que previamente era el esqueleto social del club. Esta asociación que permite el ingreso de la Sociedad Anónima se denomina "asociación soporte". Tanto la nueva Sociedad Anónima como la "asociación soporte" firman un convenio de gerenciamiento, donde se distribuyen las competencias sociales, modelo de gestión, se elaboran planes de administración (en el marco del Compliance corporativo existe un trabajo en conjunto), en general la asociación conserva todos los deportes y disciplinas no profesionales, en cambio, existe una delegación del gerenciamiento de todas las actividades deportivas profesionales a esta nueva figura societaria comercial. En términos jurídicos podríamos hablar de una cesión de derechos.

La inclusión de esta figura comercial es optativa, siempre y cuando no se presenten dos supuestos contemplados: a) La asociación participa habitualmente a la organización de manifestaciones deportivas pagas que generan beneficios superiores a 1,2 millones de euros (se toma en cuenta la media de los tres últimos ejercicios) o b) La asociación emplea deportistas cuyo monto total de remuneraciones excede 800.000 euros (se toma en cuenta la media de los tres últimos ejercicios). Esto incluye el conjunto de los salarios, primas, beneficios y vacaciones ${ }^{28}$.

Como es de observar, en general los clubes que asiduamente compiten en torneos Internacionales y firman contratos de publicidad se encontrarían alcanzados por los filtros que establece la normativa.

\section{Franquicia en el Deporte}

A continuación, analizaremos el particular modelo que presenta Estados Unidos, donde su organización, a diferencia de las estructuras tradicionales de Europa o Latinoamérica, se basa en la pertenencia a competencias autorreguladas por grupos privados de inversiones.

\subsection{Estados Unidos y un modelo de gestión y negocio auténtico}

En Estados Unidos existe un modelo de gestión basado en la franquicia deportiva muy desarrollado que ha dado paso a la expansión a los mercados internacionales. La franquicia (en este caso entendidas como un derecho exclusivo reconocido por una Liga a una empresa deportiva para operar en un marco territorial, sin que concurra la competencia de ninguna otra salvo autorización de la Liga ${ }^{29}$.

En el sistema de franquicias, el organizador de las distintas ligas deportivas -profesionales-, admite distintos participantes bajo la condición de que cumplan aspectos económicos (pago de unos cánones) para que sean sometidos a una regulación de normas específicas de esa franquicia, sin relegar equipos o con sistemas de promoción de campeonatos, como el caso que sucede en Europa y Sudamérica. Las ligas americanas no pretenden satisfacer objetivo alguno de ninguna organización superior a ellas, estas ligas surgieron fruto de la iniciativa empresarial que buscaba obtener la máxima rentabilidad económica de sus espectáculos creando un modelo de negocio. La franquicia se fundamenta en estas ligas como la concesión que otorga la Liga a una determinada sociedad, habilitándola para explotar un equipo que participe en la competición, y vinculándola a un determinado ámbito territorial -home territory-, sobre el que se otorgan determinados derechos exclusivos, a fin de asegurar la viabilidad económica del proyecto

28 Victoria-Andreu (2012), pp. 4 y 5.

29 Terol Gómez (1998), p. 49.

GARCÍA, Carlos Adolfo. Sociedades Anónimas Deportivas como modelo de gestión de clubes: viabilidad en Argentina y las experiencias en el Derecho Comparado. Revista Justicia y Derecho, Santiago, v. 2, n 1, 2019 
empresarial del -potencial- franquiciado ${ }^{30}$.

Este sistema de Franquicia es un modelo de negocio, creado en obediencia a concretar dos objetivos fundamentales: a) ganancia económica en base a concentración empresarial (ejemplos de este fenómeno son: la National Football League (NFL), la Major League Baseball (MLB), la National Basketball Association (NBA) y la National Hockey League (NHL), colaboraciones inter-empresariales); b) generación de un producto final perfecto para su comercialización (es el caso de las transmisiones televisivas de los encuentros deportivos; publicidades; exigentes estándares de calidad en las infraestructuras, conductas de los deportistas, etcétera); c) el sistema se basa en criterios de rentabilidad y estabilidad ( los méritos deportivos no son determinantes).

Para entender el modelo, es necesario graficarlo con la NBA (en inglés National Basketball Association), la principal liga de básquet fundada en Estados Unidos en el año 1946. Se trata de una entidad totalmente independiente y completamente autogestionable, que se desempeña de manera privada y al margen de la federación americana (US Basketball), y con el modelo de franquicia como principal baluarte de sus acuerdos contractuales. Así, los equipos que conforman dicha entidad -Liga-, son franquicias, que a su vez son corporaciones en sí mismas: es decir, que estamos hablando de auténticas sociedades anónimas que constituyen un gigantesco holding, en donde la Liga -NBA, una Sociedad Anónima-, está configurada como una sociedad limitada, y en la que sus socios -o propietarios-, son los equipos que operan en régimen de franquicia ${ }^{31}$.

La conformación de las ligas de los Estados Unidos se basa en condiciones establecidas por mega uniones de empresas formando grupos, donde el negocio adquiere estándares y reglas que entregan seguridad, estabilidad, claridad y lo más importante, rentabilidad al deporte en base a una equidad operativa. Como es evidente, el sistema es diferente al existente en Europa y lo es más aún al Latinoamericano. Esta marca deportiva, con su especial distribución de dividendos y ganancias, sin duda es un modelo a tener en cuenta, ya que no sólo puede ser observado a través de una óptica empresarial, sino también desde el punto de vista de administración de recursos y tecnificación y profesionalización del deporte en sí.

Ahora, cabe preguntarnos si este sistema ¿es posible implementarlo en el resto de los países del mundo?, entiendo, con cierto criterio, que la universalización de un concepto de gestión exitoso es inminente, por lo cual no veo dificultad de que así sea. En ello, existen registros donde se exponen las deficiencias de las regulaciones privadas-públicas para sostener la economía y finanzas de las organizaciones deportivas. Un caso ilustrativo de esto es el italiano, cuyo Parlamento, mediante la Ley 27 de 21 de febrero de 2003 (ley conocida como Salva Calcio), aprobó una serie de medidas fiscales y contables para clubes profesionales investigadas por la UE, por posible infracción del artículo 87 del Tratado, sobre ayudas de Estado., que llevaron al Estado aportar cantidades millonarias para la salvaguarda de la competición; otro es el de la República Argentina, donde se llevaron adelante contratos de transmisión entre el Estado Nacional y la AFA de televisación pública de los partidos de la Primera División y Campeonato de Segunda División del Fútbol Profesional, llegando a acordarse sumas multimillonarias, en orden a sostener un sistema de flujos públicos a déficit estructural de clubes $^{32}$.

30 Pérez-Serrabona González (2015).

31 Pérez-Serrabona González (2015).

32 Decreto 221/2009 (01.09.2009). Decisión Administrativa, "Créase el Programa Futbol para Todos".

GARCÍA, Carlos Adolfo. Sociedades Anónimas Deportivas como modelo de gestión de clubes: viabilidad en Argentina y las experiencias en el Derecho Comparado. Revista Justicia y Derecho, Santiago, v. 2, n 1, 2019 


\section{Cuestiones acerca de la implementación de la SAD}

Existen elementos que determinan la implementación o no de una SAD.

Como primer término entiendo que debe atenderse la materia de ingresos de la Persona Jurídica, los cuales muchas veces son reinvertidos en otros negocios, de forma tal que ellos generen ganancia para la institución. Una Sociedad puede diversificar sus actividades y materializar inversiones que generen ingresos de diferentes campos, logrando seguridad y éxito en la gestión (el caso de la mayoría de los clubes ingleses, que muchas veces son sujetos de Holdings internacionales).

El primer concepto a tener en cuenta es el extremo factor emocional que posee una estructura social como es la asociación civil, pues ella conserva para sí una identidad con sus socios, y éstos últimos ven al club bajo este formato como una entidad conservadora de principios y hasta de manejo de valores que responden a sus fundadores.

Otro elemento, y muy importante, es la responsabilidad. Generalmente la responsabilidad en las asociaciones se ve diluida en sus socios, ya que el patrimonio pertenece a todos y ante pasivos extremos que no puede enfrentar, no existe respaldo o cuanto menos la atribución de responsabilidad es difusa entre una cantidad de asociados. En cambio, en las Sociedades Anónimas, los responsables se encuentran expuestos, ya que poseen mayor o menor participación en razón de sus acciones y genera un sentido de seguridad mayor en los negocios.

Pareciera ser que la discusión debiera suceder en torno a mecanismos operativos de gestión profesional -sectorización de la persona jurídica por materia, como por ejemplo jurídica, impuestos, marketing, entre otros-, oportunidad de crecimiento y reinversión de excedentes -la figura societaria, sujeta a mayores controles, podría ampliar sus inversiones hasta en mercados financieros, desarrollando la marca a nivel global- y por supuesto evaluar el ítem de responsabilidad - la figura societaria por excelencia, enmarcada en normativas específicas, se somete a un régimen de responsabilidad más riguroso.

En general, la introducción de una figura comercial en el ámbito deportivo atraviesa discusiones una serie de debates en torno a: a) cuestiones históricas-tradicionales (participación de las familias en las actividades de un organismo sin fines comerciales y con miras a actividades recreativas y sociales, tal y como fue fundado en sus inicios); b) continuación del club (a menudo es común escuchar que las sociedades como marco jurídico, arriesga la continuidad del club, dejándolo expuesto a posibles quiebras o procesos falenciales); c) arraigados condicionamientos en torno a criterios sociológicos de "identidad" con el club (los asociados se sienten parte del club, situación que evidentemente se torna difusa si su participación se resume a ser un simple fanático que compra el ticket de partido cada fin de semana). Probablemente estos extremos deban ser abordados, y saneados a fin de que la estructura sea validada no solo en instrumentos legales que permitan su implementación sino en un estamento anterior y más estructural: aceptación social y personal del modelo, al menos a elección como ocurre en legislaciones como la francesa.

\section{Conclusión}

La realidad deportiva y de mercado en la cual participan los clubes e instituciones deportivas exige un análisis particular de cada institución entendiendo su realidad diferente, ya sea en sus ingresos, proveedores, liga en la que participa y sobre todo el ordenamiento jurídico Societario y Comercial que dispone.

GARCÍA, Carlos Adolfo. Sociedades Anónimas Deportivas como modelo de gestión de clubes: viabilidad en Argentina y las experiencias en el Derecho Comparado. Revista Justicia y Derecho, Santiago, v. 2, n 1, 2019 
El hecho de considerar a una estructura Jurídica como la ideal en cualquier ámbito es errónea, ya que cada Estado tiene una realidad diferente de mercado. No es lo mismo España o Inglaterra, que contienen clubes de gran poder económico (con una idiosincrasia determinada) que Argentina, donde los clubes deportivos nacieron con el firme propósito social de practicar deportes e incluir a la sociedad en ellos. Creo que la creación de la Superliga de Fútbol Argentina es un avance en cuanto a la gestión operativa del deporte argentino y considero que podría ser este organismo, a través de propuestas superadoras, promover la gestión corporativa de los entes deportivos (en vistas a su interés de vender el producto de televisión).

Un especial estudio debe ser hecho acerca del modelo norteamericano de franquicias deportivas. Esta vidriera de empresas conformando grupos de acción en pos de rentabilidad y estabilidad de una mega marca competitiva (entiéndase NBA, MLS por nombrar algunos) es un sistema innovador, polo de atracción de deportistas destacados de todo el mundo, con márgenes operativos positivos. La conformación de una estructura así en el viejo continente o en Sudamérica requeriría un entrenamiento cultural y empresarial, que entiendo, es necesario para hacer del deporte algo atractivo como marca.

Los debates en torno a incluir la SAD como modelo de club, debe ser en un rango optativo, es decir, posibilitando que las Asociaciones coexistan con ellas, ya sea como "soporte" (Francia) o indistintamente en uno u otro club. En el caso de Argentina, lo considero necesario, al menos que la modalidad esté a disposición, pues serán los órganos internos de los clubes quienes decidan qué sistema aplicar.

Considero que las exigencias del deporte a nivel internacional (desde el punto de vista competitivo-reglamentario o simplemente de mercado), y especialmente en el fútbol, exigen tener instalaciones de primer nivel, condiciones de logística óptimas y un plantel de jerarquía. Estas exigencias demandan grandes erogaciones, que deben ser respaldadas por una correcta administración y un efectivo control estatal. En países donde existe un mercado emergente (Argentina), donde el mercado de valores está concentrado por poca cantidad de empresas, encuentro provechosa la idea de estimular los volúmenes de activos de capitales provenientes de otros orígenes, en este caso el fútbol. De esta forma no sólo se motorizaría el mercado, sino que además obligaría a los clubes a someterse a estrictos controles por parte de la Comisión Nacional de Valores.

Al margen de la implementación o no de un tipo societario comercial en un ordenamiento jurídico, la riqueza del debate en torno a ellas es el primer paso para superar prejuicios instalados.

\section{Bibliografía Citada}

All Party Parlamentary, Football Group (2009): "English Football and its Governance, Thales". Disponible en: http://www.levelplayingfield.org.uk/sites/default/files/contentfiles/apfg_report_on_english_footbaII_its_governance_april_20091.pdf [Fecha de consulta: 01.11.2018].

Barbierl, Pablo (2011): Régimen Jurídico, Administración y Gestión de los Clubes Deportivos (Ad-Hoc, Buenos Aires).

Delgado Truyuols, Álvaro (2014): "El fracaso de las sociedades anónimas deportivas en España”. Disponible en: https://iusport.com/art/1725/el-fracaso-de-las-sociedades-anonimas-deportivas-en-espana [Fecha de consulta: 01.11.2018].

GARCÍA, Carlos Adolfo. Sociedades Anónimas Deportivas como modelo de gestión de clubes: viabilidad en Argentina y las experiencias en el Derecho Comparado. Revista Justicia y Derecho, Santiago, v. 2, n 1, 2019 
Deloitte, "Roar power, Annual Review of Football Finance 2018. Sports Business Group June 2018". Disponible en: https://www2.deloitte.com/uk/en/pages/sports-business-group/articles/annual-review-of-football-finance.html [Fecha de consulta: 29.10.2018].

González Goicochea, MARTIN E. (2018): “¿Es posible la Sociedad Anónima Deportiva en el ordenamiento argentino?". Disponible en: https://iusport.com/art/69202/es-posible-la-sociedad-anonima-deportiva-en-el-ordenamiento-argentino [Fecha de consulta: 31.10.2018].

KPMG (2018): "The European Elite 2018, Football Clubs'Valuation". Disponible en: https://home.kpmg. com/content/dam/kpmg/co/pdf/2018/06/kpmg-football-clubs-valuation-2018.pdf [Fecha de consulta: 29.10.2018].

MenÉndez, PABLO y otros (1997): "Régimen Jurídico del Futbol Profesional", Madrid.

NisSEN, Ricardo (2000): Panorama Actual De Derecho Societario (Buenos Aires, Ad-Hoc).

Olivera García, Ricardo (2001): Sociedades Anónimas Deportivas en el Derecho Uruguayo (Montevideo Uruguay, Universidad de Montevideo, Facultad de Derecho).

PÁgina WEB oficial, Salario Mínimo Colombia, 2018. Disponible en: http://www.salariominimocolombia.net/ [Fecha de consulta: 01.11.2018].

Pérez-Serrabona González, Francisco Javier (2015): "Reflexiones en torno al modus operandi de la franquicia en el deporte". Revista Internacional de Doctrina y Jurisprudencia.

The Federal Association (2015): "Club Structures". Disponible en: https://www.google.com.ar/url?sa=t\&rc$\mathrm{t}=\mathrm{j} \& \mathrm{q}=\&$ \&esrc=s\&source $=$ web\& $c d=13 \& \mathrm{cad}=\mathrm{rja} \& u a c t=8 \& \mathrm{ved}=2$ ahUKEwil5vqFIrTeAhXHFZAKHaiuDOM4ChAWMAJ6BAgGEAI\&url=http\%3A\%2F\%2Fojs.urepublicana.edu.co\%2Findex.php\%2Frevistarepublicana\%2Farticle\%2Fview\%2F1\%2F187\&usg=AOvVaw0TdGz4AmhphjM6BUwgm-NK [Fecha de consulta: 01.11.2018].

Terol Gómez, Ramón (1998): Las Ligas Profesionales (Edit. Aranzadi).

Trujlllo Cabrera, Juan (2014): "Fútbol colombiano: Conversión de Clubes en Sociedades Anónimas". Disponible en: https://www.google.com.ar/url?sa=t\&rct=j\&q=\&esrc=s\&source=web\&cd=13\&cad=rja\&uac$\mathrm{t}=8 \& \mathrm{ved}=2$ ahUKEwil5vqFIrTeAhXHFZAKHaiuDOM4ChAWMAJ6BAgGEAI\&url=http\%3A\%2F\%2Fojs. urepublicana.edu.co\%2Findex.php\%2Frevistarepublicana\%2Farticle\%2Fview\%2F1\%2F187\&usg=AOVaw0TdGz4AmhphjM6BUwgm-NK [Fecha de consulta: 01.11.2018].

VeIGA, Gustavo (2016): "Fútbol Argentino Sociedad Anónima". Disponible en: https://www.pagina12.com. ar/diario/deportes/8-307947-2016-08-28.html [Fecha de consulta: 31.10.2018].

Victoria Andreu, Francisco (2012): "Asociación versus Sociedad Anónima Deportiva”. Disponible en: http:// www.iusport.es/images/stories/autores/Francisco-Victoria-Sociedades-Deportivas-FVA2012.pdf [Fecha de consulta: 24.05.2018].

W.AA. (2016): "El fútbol en la economía Argentina". Disponible en: https://arklems.files.wordpress. com/2011/05/coremberg-2016-el-fc3batbol-en-la-economc3ada-argentina.pdf [Fecha de consulta: 22.07.2018]. 


\section{NORMAS CITADAS}

ASOCIACIÓN DEL FUTBOL ARGENTINO, Estatuto, Ciudad Autónoma de Buenos Aires, 2017. Disponible en: http://www.afa.com.ar/upload/reglamento/Estatuto\%20AFA\%20-\%20Desde\%2024.02.2017.pdf [Fecha de consulta: 23.07.2018].

Cfr. CONGRESO DE LA REPUBLICA DE COLOMBIA (2011): Ley Nacional de Colombia No 1445, Bogotá. Arts. 1, 3, 5, 8, 9, 10 y ss. Disponible en: http://www.coldeportes.gov.co/normatividad/normatividad_juridica/ leyes/ley_1445_2011 [Fecha de consulta: 05.07.2018].

Código Civil y Comercial de La Nación Argentina, edición 2015.

CONGRESO NACIONAL DE CHILE, Ley No 20.019 de Sociedades Anónimas Deportivas Profesionales. Santiago de Chile, 2005.

Decreto 221/2009, Decisión Administrativa, "Créase el Programa Futbol para Todos”, 2009.

FEDERACION INTERNACIONAL DE FÚTBOL ASOCIADOS, Reglamento sobre el Estatuto y la Transferencia de los Jugadores de Fútbol, Suiza, 2018. Disponible en: https://resources.fifa.com/image/upload/regulations-on-the-status-and-transfer-of-players.pdf?cloudid=n0i87huagfrosp7pgrhz [Fecha de consulta: 23.07.2018].

Ley No 17.292, Montevideo, Uruguay. “Administración Pública, Empleo, Fomento y Mejoras", 2001.

Ley No 29.504, Lima, Perú. Ley que promueve la transformación y participación de los Clubes Deportivos de fútbol profesional en Sociedades Anónimas Abiertas, 2010. 